\title{
On the development of an apatitic calcium phosphate bone cement
}

\author{
MANOJ KOMATH*, H K VARMA and R SIVAKUMAR \\ Bioceramics Division, Biomedical Technology Wing, Sree Chitra Tirunal Institute for Medical Sciences and \\ Technology, Thiruvananthapuram 695 012, India
}

MS received 29 October 1999; revised 9 February 2000

\begin{abstract}
Development of an apatitic calcium phosphate bone cement is reported. $100 \mu$ Particles of tetracalcium phosphate (TTCP) and dicalcium phosphate dihydrate (DCPD) were mixed in equimolar ratio to form the cement powder. The wetting medium used was distilled water with $\mathrm{Na}_{2} \mathrm{HPO}_{4}$ as accelerator to manipulate the setting time. The cement powder, on wetting with the medium, formed a workable putty. The setting times of the putty were measured using a Vicat type apparatus and the compressive strength was determined with a Universal Testing Machine. The nature of the precipitated cement was analyzed through X-ray diffraction (XRD), fourier transform infrared spectrometry (FTIR) and energy dispersive electron microprobe (EDAX). The results showed the phase to be apatitic with a calcium-to-phosphorous ratio close to that of hydroxyapatite. The microstructure analysis using scanning electron microscopy (SEM) showed hydroxyapatite nanocrystallite growth over particulate matrix surface. The structure has an apparent porosity of $\sim 52 \%$. There were no appreciable dimensional or thermal changes during setting. The cement passed the in vitro toxicological screening (cytotoxicity and haemolysis) tests. Optimization of the cement was done by manipulating the accelerator concentration so that the setting time, hardening time and the compressive strength had clinically relevant values.
\end{abstract}

Keywords. Calcium phosphate cements; tetracalcium phosphate; hydroxyapatite; bioceramics; bone substitute.

\section{Introduction}

The advent of calcium phosphate bone cements (CPBC) is considered as a remarkable development in the field of bone repair materials. CPBCs offer a route of obtaining orthocalcium phosphates in a monolithic form at physiological conditions, without sintering process, by means of a cementitious reaction.

Generally, a CPBC formulation contains calcium and phosphorous based ingredients in powder form, which on mixing with an aqueous medium forms a workable, selfsetting putty. The ingredients dissolve in the medium making it supersaturated with a desired calcium phosphate which gets reprecipitated inside the mass. The growth of the calcium phosphate phase as entangled crystallites, helps the putty to retain its strength and shape (Driessens et al 1995).

The design of a CPBC is done on the basis of the calcium-phosphorous $(\mathrm{Ca} / \mathrm{P})$ ratio of the final precipitate and the physicochemical characteristics of precipitation (setting time, hardness, chemical stability, biofunctionality etc). Atleast two reactants are necessary, with differing $\mathrm{Ca} / \mathrm{P}$ ratios adjusted to obtain the desired phase. A list of reactants used in successful formulations, is given in

\footnotetext{
*Author for correspondence
}

table 1. The wetting medium essentially contains water along with compounds for manipulating the setting time (accelerators or retarders). The precipitating phase can be one among those given in table 2. Additives are often incorporated to enhance the properties and to promote bioactivity. Substituted phases like carbonate apatite or fluoroapatite can also be precipitated by adding suitable compounds in the cement mix (Driessens et al 1995).

The CPBCs have a distinct advantage of biocompatibility over other bone cements used for filling and fixing applications. Acrylic (PMMA) based cements pose the risk of monomer toxicity, glass-ionomer cements have the problem of metallic component leach out and bioactive bone cements have the drawback of poor bio-degradability. In the case of CPBCs, the final precipitate is a calcium orthophosphate and hence, non-toxicity can be assured (Driessens et al 1995, 1998).

The ceramic forms of orthocalcium phosphates have become materials of choice for bone repair, because of their bio-compatibility and osteoconductivity. However, bioceramics in conventional forms cannot be instantly molded into a desired shape, which is the most crucial requirement in orthopedic application. The granule form of bioceramics supplied for filling applications, may tend to drift away from the site of implantation. CPBCs are attractive as they are mouldable. The cement can be shaped into any complicated geometry or filled into any 
intricate cavity, within its setting period. It can adapt to the bone cavity, offering a good fixation and optimum tissue-biomaterial contact necessary for stimulating bone ingrowth (Driessens et al 1995).

Another attractive feature of CPBCs is that it can be made into an injectable form, which simplifies the bone repair procedures from major surgery to a key-hole operation. The investigation in this direction seems promising (Constantz et al 1995; Knaack et al 1998). Many other possibilities like drug delivery and growth factor incorporation are under investigation (Driessens et al 1998).

In this paper, we report the development of a calcium phosphate bone cement suitable for orthopedic applications, with TTCP and DCPD as ingredients. The design of the cement, physico-chemical evaluation, toxicological screening and optimization for the proposed application, are presented in subsequent sections.

\section{Experimental}

\subsection{Preparation of materials}

Analar grade dicalcium phosphate dihydrate (DCPD) was used which was ball milled to reduce the particle sizes to below $100 \mu \mathrm{m}$. TTCP was synthesized in house, out of $\mathrm{CaHPO}_{4}$ and $\mathrm{CaCO}_{3}$ in a solid state reaction process given below.

The reactant powders (dicalcium phosphate and calcium carbonate) were mixed in equimolar ratio and subjected to milling, to have a uniform particle size and even distribution. The powder was fired at $1350^{\circ} \mathrm{C}$ for $3 \mathrm{~h}$ in a platinum crucible and rapidly cooled to room temperature. The product phase was identified to be pure in X-ray diffraction analysis (JCPDS Card No: 25-1137). The lump of TTCP obtained after cooling, was crushed to a particle size $<100 \mu \mathrm{m}$.

\subsection{Formulation of the cement}

The cement powder was prepared by mixing TTCP and DCPD particles thoroughly in equimolar ratio. It was then stored in dry and sterile conditions. The liquid medium was prepared with double distilled demineralised water. The wetting ratio, i.e. the amount of the medium to be added to the cement, was decided on the basis of workability of the putty formed.

The cement was observed to form a workable putty only in a narrow range of wetting ratio. Insufficient amount of the medium will make the cement powder an inhomogeneous mass, with which shaping is difficult. Excessive amount of medium, on the other hand, will give a loose paste. For the proposed application, a putty that can be shaped with fingers will be ideal. The optimum wetting to obtain such a form, was found to be $0.5 \mathrm{ml}$ of the medium per $1 \mathrm{~g}$ of cement powder. This wetting ratio was followed throughout the study for setting the cement.

The long setting periods (> $60 \mathrm{~min}$ ) normally observed in TTCP + DCPD cements (Driessens et al 1993) make it necessary to add appropriate chemicals for accelerating the setting. Disodium hydrogen phosphate $\left(\mathrm{Na}_{2} \mathrm{HPO}_{4}\right)$ which satisfy the biocompatibility requirements was selected as the accelerator (Fulmer and Brown 1993; Ginebra et al 1995).

\subsection{Property evaluation}

The setting times of the cement were assessed using a Vicat type apparatus (Philips 1991). It consisted of a steel

Table 1. The reactants used in successful calcium phosphate bone cement formulations listed according to their calcium-to-phosphorous ratio.

\begin{tabular}{lllc}
\hline Compound & Abbreviation & \multicolumn{1}{c}{ Formula } & Ca/P ratio \\
\hline Phosphoric acid, anhydrous & & $\mathrm{H}_{3} \mathrm{PO}_{4}$ & 0 \\
Monocalcium phosphate, anhydrous & MCPA & $\mathrm{Ca}\left(\mathrm{H}_{2} \mathrm{PO}_{4}\right)_{2}$ & $0 \cdot 5$ \\
Dicalcium phosphate, anhydrous & DCPA & $\mathrm{CaHPO}$ & $1 \cdot 0$ \\
Dicalcium phosphate dihydrate & DCPD & $\mathrm{CaHPO}_{4} \cdot 2 \mathrm{H}_{2} \mathrm{O}$ & $1 \cdot 0$ \\
Tricalcium phosphate (alpha and beta) & $\alpha / \beta-\mathrm{TCP}$ & $\mathrm{Ca}_{3}\left(\mathrm{PO}_{4}\right)_{2}$ & $1 \cdot 5$ \\
Tetracalcium phosphate & TTCP & $\mathrm{Ca}_{4}\left(\mathrm{PO}_{4}\right)_{2} \mathrm{O}$ & $2 \cdot 0$ \\
Calcium oxide/hydroxide & & $\mathrm{CaO} / \mathrm{Ca}(\mathrm{OH})_{2}$ & $\infty$ \\
\hline
\end{tabular}

Table 2. The precipitates formed in the setting cement. The phases formed will depend on the nature of the reactants and the aggregate ratio of calcium and phosphorous in the mix.

\begin{tabular}{lclc}
\hline Compound & Abbreviation & \multicolumn{1}{c}{ Formula } & Ca/P ratio \\
\hline Dicalcium phosphate dihydrate & DCPD & $\mathrm{CaHPO}_{4} \cdot 2 \mathrm{H}_{2} \mathrm{O}$ & $1 \cdot 00$ \\
Octacalcium phosphate & $\mathrm{OCP}$ & $\mathrm{Ca}_{8} \mathrm{H}_{2}\left(\mathrm{PO}_{4}\right)_{6} \cdot 5 \mathrm{H}_{2} \mathrm{O}$ & $1 \cdot 33$ \\
Amorphous calcium phosphate & ACP & $\mathrm{Ca}_{3}\left(\mathrm{PO}_{4}\right)_{2} \cdot 3 \mathrm{H}_{2} \mathrm{O}$ & $1 \cdot 50$ \\
Hydroxyapatite & $\mathrm{HA}$ & $\mathrm{Ca}_{10}\left(\mathrm{PO}_{4}\right)_{6}(\mathrm{OH})_{2}$ & $1 \cdot 67$ \\
\hline
\end{tabular}


needle (diameter $1 \mathrm{~mm}$ ) moving along a vertical axis under a constant load of $100 \mathrm{gf}$, and a plastic well of $5 \mathrm{~mm}$ depth to hold the putty. The cement was mixed and filled in the well and the needle was allowed to penetrate into it. As the mass solidifies, the penetration depth decreases. The distance traveled by the tip can be measured to $0.01 \mathrm{~mm}$ accuracy using an attached dial gauge. Measurements were repeated at time intervals of $30 \mathrm{sec}$.

There are two stages identified in the setting of $\mathrm{CPBCs}$ - the initial setting time which denotes the end of workability of the putty after wetting, and the final setting time which indicates the hardening of the set mass (Driessens et al 1995). The initial setting time was measured by monitoring the penetration of a blunt needle. The needle can no more penetrate after the initial setting. Thereafter, a needle with a sharp tip was used on the set mass to see the final setting. The tip cracks the putty and enters into the surface region till the mass hardens.

Compressive strength of the set cement was measured in an Instron Universal Testing Machine. The cement samples were molded in the form of cylindrical pellets (6 $\mathrm{mm}$ dia and $12 \mathrm{~mm}$ height), incubated in 100\% humidity for $24 \mathrm{~h}$ and dried before the analysis. The pellets were compressed along their height in between the platens of the machine at a cross-head speed of $1 \mathrm{~mm} / \mathrm{min}$. The compressive strength was calculated from the break load and dimensions of the pellets.

The phase analysis of the set cement was carried out using X-ray diffraction (XRD) technique. The set cement mass was kept in $100 \%$ humidity at physiological conditions for $24 \mathrm{~h}$ and then dried and powdered for analysis. FTIR spectrometry was also done by $\mathrm{KBr}$ pellet technique, after powdering the set cement. The $\mathrm{Ca} / \mathrm{P}$ ratio was determined using microprobe energy dispersive analysis (EDAX). The microstructure of the cement was observed in scanning electron microscope (SEM), after cutting the set mass across and polishing. The porosity was found by measuring the density of the cement mass and comparing it with the theoretical density of the phase. The change in dimensions during setting was assessed using standard moulds. The setting temperature was monitored in a differential thermal analyzer (DTA) in isothermal mode with alumina as the standard.

\subsection{Toxicological evaluation}

The optimized cement was subjected to cytotoxicity and haemolysis screenings following the ISO/ASTM protocols. The samples were set in the form of pellets and preserved at $100 \%$ humidity for $24 \mathrm{~h}$ at physiological conditions, prior to the analyses. Sterilization was done by autoclaving at $393 \mathrm{~K}$ for $20 \mathrm{~min}$.

The cytotoxicity studies were carried out with mouse fibroblast cells (L929). The medium used was Eagle's MEM with $10 \%$ foetal bovine serum. The cells were sub- cultured into monolayers and the samples were placed (in duplicate) over the cells. After an incubation of $24 \mathrm{~h}$ at $310 \mathrm{~K}$ under $5 \% \mathrm{CO}_{2}$, the cell morphology was analyzed under a phase contrast microscope.

The haemolytic potential of the cement was tested using fresh rabbit blood with potassium oxalate as anticoagulant. The sterilized cement pellets (5 numbers, each weighing $0.5 \mathrm{~g}$ ) were prepared in $5 \mathrm{ml}$ normal saline and incubated with $0.1 \mathrm{ml}$ blood for $90 \mathrm{~min}$ at $37^{\circ} \mathrm{C}$. The haemolysis was assessed after centrifuging, using a spectrophotometer, in comparison with the controls.

\section{Results and discussion}

\subsection{Characteristics of the cement}

3.1a Setting time: A known quantity of the cement powder was taken and wetted with the medium in a ratio $0.5 \mathrm{ml} / \mathrm{g}$. After mixing for $30 \mathrm{sec}$, the putty was loaded in the apparatus for measurements.

Distilled water as the wetting medium took longer time to set-more than $60 \mathrm{~min}$. Addition of the accelerator, disodium hydrogen phosphate $\left(\mathrm{Na}_{2} \mathrm{HPO}_{4}\right)$, at the concentration of $0 \cdot 1 \mathrm{M}$ in the medium showed a marked effect that the initial setting time came down to $15 \mathrm{~min}$. At higher concentrations, the initial and final setting times decreased as given in the plot (figure 1).

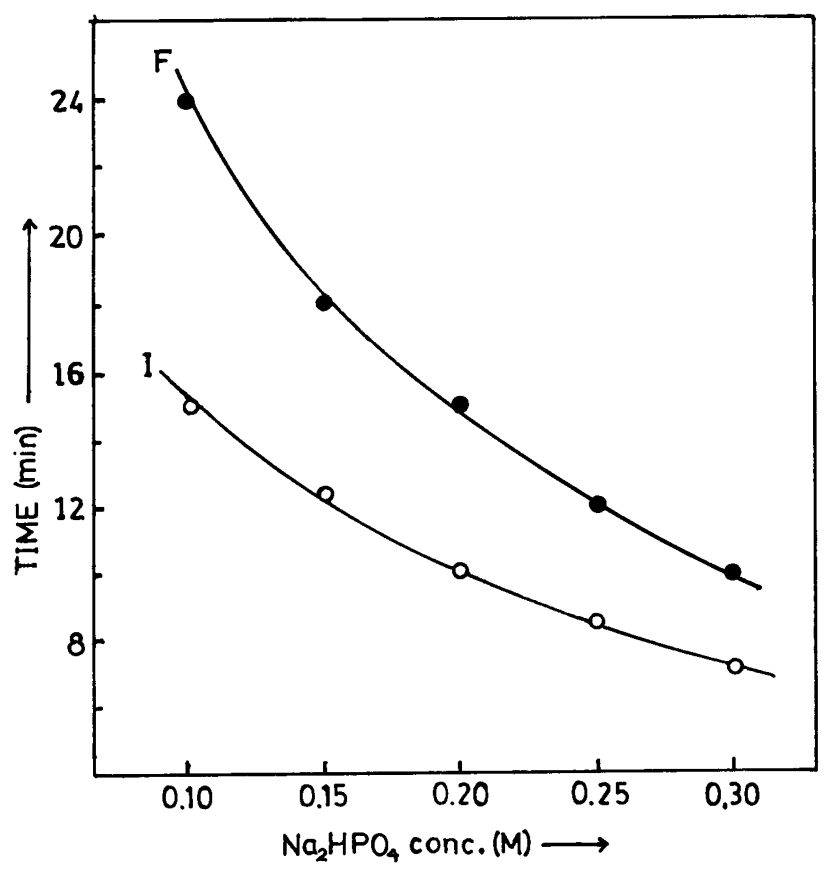

Figure 1. The setting times of the cement with a constant wetting ratio of $0.5 \mathrm{ml} / \mathrm{g}$ at different concentrations of $\mathrm{Na}_{2} \mathrm{HPO}_{4}$ in the medium. Curve I (marked with 'o') and curve F (marked with ' $\bullet$ ') denote the initial and final setting times, respectively. 
3.1b Compressive strength: Among the mechanical properties of bone repair materials, compressive strength (CS) is the most widely explored one. The strength of a cement depends upon various factors like particle size of the cement powder, nature of additives and wetting ratio. The effect of concentration of accelerator in the medium was investigated in order to optimize the cement.

The range of CS obtained (10-12 MPa) was found comparable to reported values for calcium phosphate cements (Driessens et al 1993, 1998). The effect of the accelerator $\left(\mathrm{Na}_{2} \mathrm{HPO}_{4}\right)$ concentration on the CS was studied by keeping the wetting ratio constant. The results are shown in figure 2. A linear decrease in CS with the increase in concentration, can be observed.

3.1c Precipitated phase: The calcium phosphate phase formed in the cement (set with $0.2 \mathrm{M} \mathrm{Na}_{2} \mathrm{HPO}_{4}$ in the medium at a wetting ratio $0.5 \mathrm{ml} / \mathrm{g}$ ) was analyzed in XRD. The diffraction pattern of the raw powder and that of the set cement (kept for $24 \mathrm{~h}$ in $100 \%$ humidity) are shown in figures $3 \mathrm{a}$ and $3 \mathrm{~b}$, respectively. The peaks corresponding to hydroxyapatite (HA) can be seen prominently in the cement (JCPDS Card No: 9-432). The broadness of the HA peaks indicates that the precipitated material is not well-crystallized. The peaks of DCPD were totally absent in the set cement. Traces of TTCP, however, were still present. This is due to the incomplete conversion of the compound owing to the rate limiting nature of dissolution (Tenhueisen and Brown 1996).

The $\mathrm{Ca} / \mathrm{P}$ ratio determined through EDAX is 1.69 , which is close to the theoretical value for HA (1.67).

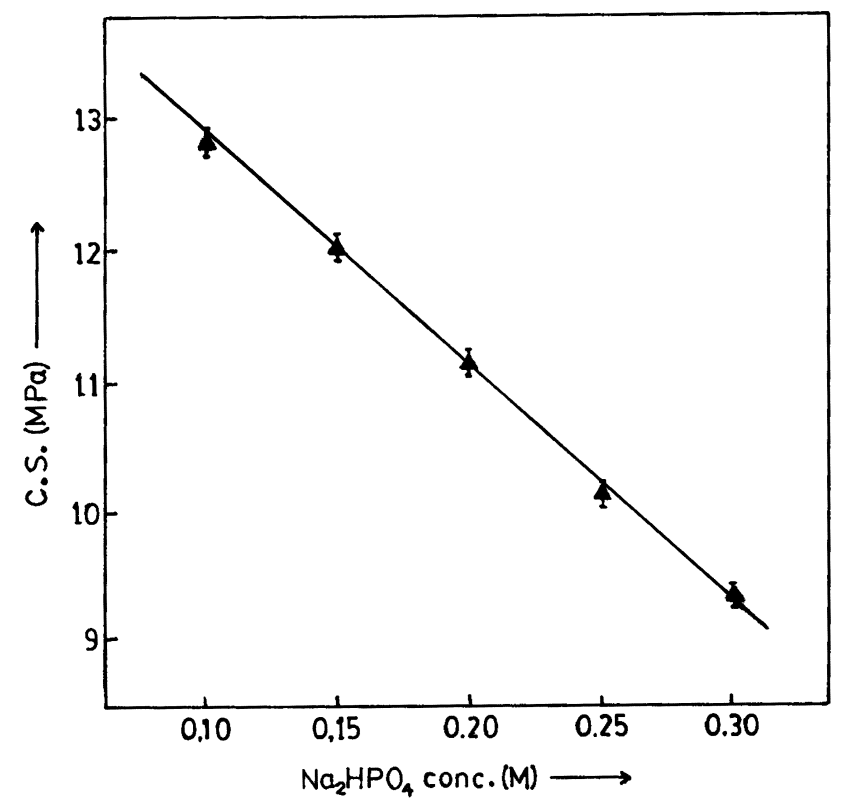

Figure 2. Compressive strength (mean values with standard deviation) of the cement at different concentrations of $\mathrm{Na}_{2} \mathrm{HPO}_{4}$ in the medium.
The FTIR of the above sample ( $\mathrm{KBr}$ pellet method) is shown in figure 4 , in which typical absorption bands corresponding to hydroxyapatite are seen (Rehman and Bonfield 1995). Trace amounts of carbonate showed up in the spectrum, which may be a result of atmospheric $\mathrm{CO}_{2}$ contamination. (Carbonate group in CPBCs is not considered as an impurity because the bone material is basically a $-\mathrm{CO}_{3}$ substituted hydroxyapatite).

3.1d Microstructure and porosity: The scanning electron micrograph of the cut face of set cement (kept in $100 \%$ humidity for $24 \mathrm{~h}$ at $37^{\circ} \mathrm{C}$ ) is shown in figure 5 . The micromorphology shows lumps of particles, interconnected in the form of porous structure. Apatitic nano-

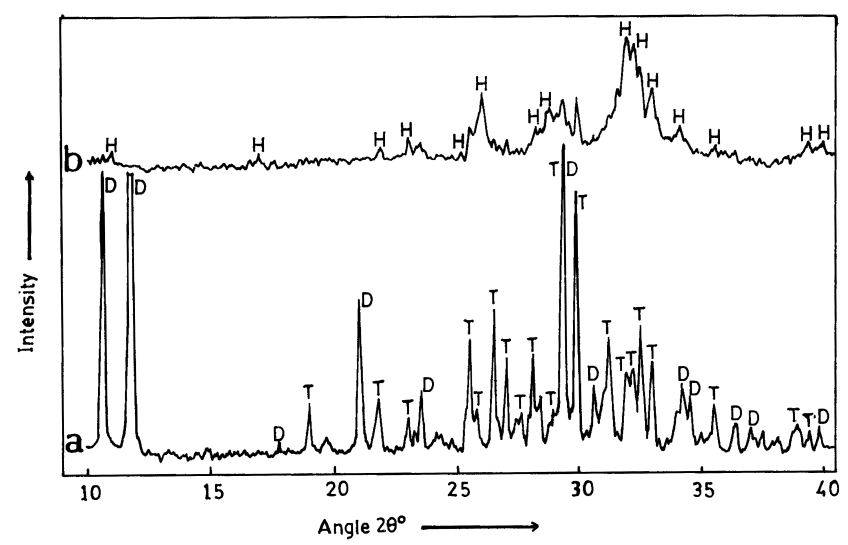

Figure 3. The XRD pattern of the raw powder (a) and that of the set cement (b). Peaks corresponding to hydroxyapatite, tetracalcium phosphate and dicalcium phosphate dihydrate are denoted as $\mathrm{H}, \mathrm{T}$ and $\mathrm{D}$, respectively.

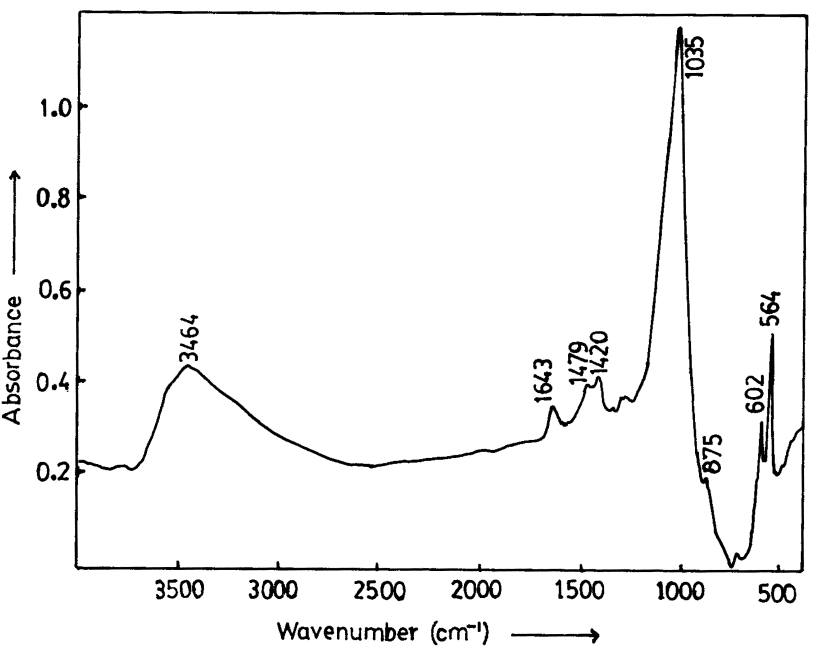

Figure 4. FTIR absorbance spectrum of the cement sample. The peak assignments for wave numbers are as follows: band around 3464, hydroxyl stretch; 1643, hydroxyl $v_{3} ; 1479$ and 1420 , carbonate $v_{3} ; 1035$, phosphate $v_{3} ; 875$, carbonate $v_{2} ; 602$ and 564, phosphate $v_{4}$ (refer Rehman and Bonfield 1995). 
crystallites can be seen grown all over the surfaces of the matrix. These appear to be heterogeneously nucleated over the surfaces of the reactant particles, as discussed by Brown and Fulmer (1991).

The density of the cement material was found to be $1.52 \mathrm{~g} / \mathrm{cc}$ from the mass-volume relationship. A comparison with the theoretical density $(3.16 \mathrm{~g} / \mathrm{cc})$ of HA, gave $\sim 52 \%$ gross porosity value.

3.1e Shrinkage: Bone cement shrinkage during setting is one of the problems encountered in bone repair when acrylic cements are used (Driessens et al 1995). The shrinkage of the TTCP + DCPD cement was assessed by setting the cement inside dies of regular geometry and known dimension. The set cement preserved in $100 \%$ humidity did not show any linear shrinkage at an accuracy level of $\pm 0.05 \mathrm{~mm}$. The set cement, on drying up, showed an average linear shrinkage of $0 \cdot 2 \%$.

3.1f Thermal changes: Observations on the setting temperature of the cement in DTA showed a decrease of $\sim 1^{\circ} \mathrm{C}$ with respect to the reference sample of same weight, at isothermal conditions. This can be compared with the exothermic setting reaction of classic acrylic bone cements, which cause a rise in the local temperature to a value nearing $100^{\circ} \mathrm{C}$ (Driessens et al 1995).

\subsection{Optimization of the cement for orthopedic application}

The results establish a route to obtain a self-setting calcium phosphate cement of apatitic nature from TTCP + DCPD formulation. The characteristics of the cement (handling properties, setting time, strength, microstructure etc) depend upon the factors like particle size, wetting ratio and wetting medium composition. The properties of the cement are to be manipulated and optimized for a particular application.

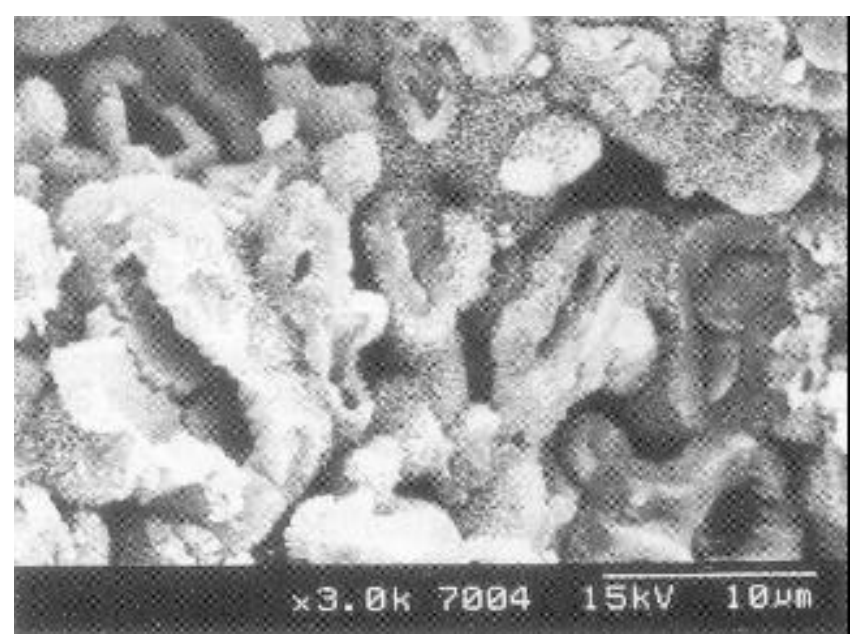

Figure 5. SEM picture of the set cement.
The handling properties of an aqueous cement are mainly decided by the particle sizes and the wetting ratio. The particle sizes of the ingredients (TTCP + DCPD) are already fixed to $50-100 \mu$ range, to have a putty consistency on wetting. The wetting ratio is selected to be $0.5 \mathrm{ml} / \mathrm{g}$ considering the workability, as described in $\S 2.2$.

The setting characteristics and strength of the cement are controlled by the amount of accelerator added. The accelerator becomes inevitable because the inherent setting time of TTCP + DCPD combination is unacceptably long. The slow precipitation is due to the rate limiting nature of dissolution of the reactants which prevents the supersaturation of $\mathrm{PO}_{4}$ ions. The function of the accelerator is to impart $\mathrm{PO}_{4}$ ions to the medium to create sufficient supersaturation. This speeds up the precipitation reaction, thereby bringing down the setting time. The effect of disodium hydrogen phosphate $\left(\mathrm{Na}_{2} \mathrm{HPO}_{4}\right)$ addition on the initial and final setting times of the TTCP + DCPD cement can be seen in figure 1 .

The manipulation of the setting times of CPBCs is significant as they should meet the requirements of surgical procedures. The initial setting time should be adjusted so as to allow sufficient time gap for shaping and filling. After the filling, it is not advisable to disturb the set cement till its hardening because any mechanical strain during this period will produce cracks and adversely affect the strength. Therefore it requires a shortest possible final setting time so that the wound closure is not delayed.

An initial setting time of about 8 min and a final setting time of $<15 \mathrm{~min}$ are recommended for orthopedic applications (Driessens et al 1998). However, bringing down the setting times by the addition of the accelerator affects the mechanical strength of the cement adversely, as evident from the CS data (figure 2). The mechanical requirement of the cement intended for filling non-loadbearing areas is such that the compressive strength should be comparable to that of human trabecular bone, i.e. not less than $10 \mathrm{MPa}$.

Thus, for optimizing the cement, the accelerator concentration has to be selected judiciously, taking the setting time and mechanical strength into consideration. Following the data represented in figures 1 and 2 , it was found that $\mathrm{Na}_{2} \mathrm{HPO}_{4}$ concentration of $0.2 \mathrm{M}$ in the medium would make the cement ideal for orthopedic filling applications. The initial and final setting times of the cement at this concentration are $10 \mathrm{~min}$ and $15 \mathrm{~min}$, respectively and the corresponding compressive strength is $11 \cdot 15 \mathrm{MPa}$.

\subsection{Biocompatibility screening}

3.3a Cytotoxicity: The cells in contact with the pellets showed the normal morphology as the cells cultured without any sample, proving that the cement is not toxic to cells. 
3.3b Haemolysis: The percentage of haemolysis due to the cement was found to be 1.58 which is well below the toxicity range.

\section{Conclusion}

A apatitic calcium phosphate bone cement formulation was developed with tetracalcium phosphate (TTCP) and dicalcium phosphate dihydrate (DCPD) particulate mixture. An aqueous medium containing $\mathrm{Na}_{2} \mathrm{HPO}_{4}$ as accelerator was provided to make it a self-setting, workable putty. Studies on the setting characteristics and the compressive strength showed that the properties were within the range of interest.

The various analyses revealed that the solidified putty is apatitic with porous morphology. The cement was found free from problems like shrinkage and setting exotherm. The set cement material passed the in vitro toxicological screening (cytotoxicity and haemolysis) tests.

The optimization of the cement for orthopedic filling application was done by adjusting the concentration of the accelerator. It was found that the setting time and hardening time decreased on increasing the accelerator concentration, but it had a deteriorating effect on the compressive strength. The optimum concentration of $\mathrm{Na}_{2} \mathrm{HPO}_{4}$ was decided to be $0.2 \mathrm{M}$, compromising setting properties and strength. At this value, the setting and hardening times (10 and $15 \mathrm{~min}$, respectively) and compressive strength (11.15 MPa) satisfy the clinical requirements of filling.

\section{Acknowledgements}

Thanks are due to Dr P V Mohnan, Dr T V Kumari, Mr $\mathrm{R}$ Sreekumar and Mrs C Radhakumari for conducting the various tests and analyses. The authors thank their colleagues in the lab for their help during this work.

\section{References}

Brown P W and Fulmer M T 1991 J. Am. Ceram. Soc. 74934

Constantz B R et al 1995 Science 2671796

Driessens F C M, Boltong M G, Bermudez O and Planell J A 1993 J. Mater. Sci.: Mater. Med. 4503

Driessens F C M, Planell J A and Gil F J 1995 in Encyclopedic handbook of biomaterials and bioengineering, Part B (New York: Marcel Dekker Inc) Vol. 2 p 855

Driessens F C M, Planell J A, Boltong M G, Khairoun I and Ginebra M P 1998 J. Eng. Med. (Proceedings of the Institution of Mechanical Engineers, Part H) 212427

Fulmer M T and Brown P W 1993 J. Biomed. Mater. Res. 27 1095

Ginebra M P, Boltong M G, Fernandez E, Planell J A and Driessens F C M 1995 J. Mater. Sci.: Mater. Med. 6612

Knaack D, Goad M E P, Aiolova M, Rey C, Tofighi A, Chakravarthy P and Lee D D 1998 J. Biomed. Mater. Res. 43399

Philips R W 1991 in Science of dental materials (Philadelphia: W B Saunders Co) 9 ed. p 74

Rehman I and Bonfield W 1995 in Bioceramics (Oxford: Elsevier Science) Vol. 8 pp 163

Tenhueisen K S and Brown P W 1996 J. Mater. Sci.: Mater. Med. 7307 